\title{
Satisfacción con la vida, comunicación con padres y estrés percibido en jóvenes universitarios del Noreste de México
}

\section{Satisfaction with life, communication with parents and perceived stress in college students from Northeast Mexico}

\author{
Rodrigo Cantú1, Julymar Alegre, Oswaldo Martínez, Mayra Chávez, \\ Sissi Arellano, Cesar Saucedo, Javier Talamantes \& René Landero \\ Facultad de Psicología, Universidad Autónoma de Nuevo León, México
}

(Recepción: Junio 2010 - Aceptación: Octubre 2010)

\begin{abstract}
Resumen
En este estudio se procedió a determinar las diferencias y relaciones existentes entre los niveles de estrés percibido, la satisfacción con la vida y la comunicación con el padre y con la madre, entre 482 jóvenes estudiantes de licenciatura en psicología de tres ciudades del noreste de México (Monterrey, Tampico y Saltillo). Se aplicaron escalas con índices adecuados de confiabilidad. En las ciudades estudiadas, se encontró que a mayor comunicación con los padres mayor satisfacción con la vida, asimismo se encontraron diferencias en los niveles de estrés percibido y satisfacción con la vida en población femenina y una correlación significativa negativa general entre estrés percibido y satisfacción con la vida. Se encuentran algunas similitudes generales en esta población del noreste de México, por lo que recomendamos realizar más estudios de comparación con población de otras áreas del sur y centro del país.

Palabras claves: Satisfacción con la vida, estrés percibido, comunicación con padres, jóvenes estudiantes mexicanos.
\end{abstract}

\begin{abstract}
In this study we proceeded to determine the differences and relationships between perceived stress levels, satisfaction with life and existing communication with the father and mother, among 482 young undergraduate students of psychology in three cities in the Northeast Mexico (Monterrey, Tampico and Saltillo). Scales were applied with adequate rates of reliability. In the cities studied, we found that increased communication with parents greater life satisfaction, were also found differences in levels of perceived stress and life satisfaction in female population and a significant negative correlation between perceived stress and general satisfaction with life. There are some general similarities in this population of northeastern Mexico, and we recommend further studies of population compared with other areas of southern and center of Mexico.
\end{abstract}

Key words: satisfaction with life, perceived stress, communication with parents, Mexican college students.

\section{Introducción}

Se le denomina jóvenes mayores a las personas que se encuentran en edades que oscilan entre los 17 y 25 años, si bien este grupo podría corresponder generalmente a quienes cursan la educación

\footnotetext{
1 Correspondencia dirigida a: Rodrigo Cantú. Facultad de Psicología, Universidad Autónoma de Nuevo León, México. Mutualismo 110, Col. Mitras Centro, C.P. 64460 Monterrey, N.L., México. E-Mail: rodrigocantu@yahoo.com.
} 
superior. En este periodo se espera que existan demandas y cambios en los roles más orientados hacia la responsabilidad así como también a tener una expectativa de vida mediante una profesión. A la par de estas demandas existen diversas variables que pueden influir en su desempeño y la motivación, tales como el qué tan satisfechos están, cómo manejan las situaciones difíciles y qué tanto apoyo tienen ya sea en la familia u otros grupos de relevancia. De esta manera se pretende estudiar la dinámica de variables como estrés percibido, satisfacción con la vida y comunicación con padres en población de tres ciudades del noreste de México.

Se define satisfacción con la vida como una evaluación cognitiva de la calidad de las experiencias propias mediante un indicador del bienestar subjetivo de la propia persona, un sentimiento de bienestar que difiere de la aprobación o desaprobación de las condiciones objetivas de la vida.

Este elemento forma parte del constructo bienestar subjetivo que es el sentimiento de bienestar en relación con uno mismo, esto es sentirse bien (subjetivo) y no necesariamente estar bien (objetivo), surge de las relaciones del individuo con su entorno familiar y social, en donde se incluyen las condiciones de vida materiales y sociales que ofrecen al hombre determinadas oportunidades para su realización personal (Arita-Watanabe, 2005; Montoya \& Landero, 2008).

Otros autores agregan que la satisfacción sería un estado psicológico resultante de la transacción entre el individuo (personalidad) y su entorno micro social (estado civil, familia, participación social, satisfacción laboral, apoyo social) y macro social (ingresos, cultura) (García-Viniegras \& González, 2000).

Ardila (2003) propone una definición integradora del concepto al establecer que la calidad de vida es un estado de satisfacción general, derivado de la realización de las potencialidades de las personas. Es una sensación subjetiva de bienestar físico, psicológico y social, que incluye en lo subjetivo la intimidad, la expresión emocional, productividad personal, seguridad y salud percibida. Como aspectos objetivos el bienestar material, las relaciones armónicas con el ambiente físico, social, con la comunidad y la salud objetivamente percibida.

Diener y Eunkook (2003) argumentan que nuestro país puntúa más alto en satisfacción con la vida sobre países como Francia y España, a pesar de que sus indicadores de bienestar económico son más altos. Por otra parte en estudios con población española y argentina se ha encontrado una tendencia a percibir una insatisfacción generalizada en las ciudades más densamente pobladas, por el contrario en ciudades de menor densidad se encuentra una mayor satisfacción (Castro-Solano \& Díaz-Morales, 2002).

Por otro lado, Moyano y Ramos (2007), establecen que el estado civil, también ha mostrado diferencias al reportar que las personas solas -solteras y viudas, entre otras- son menos felices que las casadas y, que la satisfacción con la vida va en aumento conforme aumenta la edad (Argyle, 1992). $\mathrm{Al}$ respecto, Moyano y Ramos (2007) concluyen que los jóvenes mayores (17-25 años) mostraban una mayor dificultad para alcanzar niveles importantes de satisfacción vital debidos a sus elevadas expectativas de vida y sus actuales recursos para alcanzar metas a futuro, además de su reciente salida de la adolescencia como un proceso conflictivo de la vida.

Otro aspecto que también parece influir es el tipo de familia, así lo demuestran los resultados obtenidos por Montoya y Landero, (2008), en su estudio realizado con jóvenes universitarios, donde se observó que el hecho de convivir en una familia con ambos padres permite una mayor autoestima y satisfacción con la vida reportándose en estos jóvenes mejor calidad de vida.

Como se ha mencionado, la satisfacción con la vida surge de las relaciones del individuo con su entorno familiar y social. Lo mismo ocurre en su influencia con la comunicación. En este sentido cabe resaltar que la familia, como la más antigua y básica de las instituciones humanas tiene a su cargo la transmisión de las normas, roles y creencias propios de la cultura vigente (Linton, 1972), así como también cubre la función de formar la personalidad e identidad de los individuos que de ella dependen, que permitirán la expresión de afecto, la capacidad de decisión y la negociación circunstancial de problemas, brindándoles los elementos necesarios para sobrevivir y adaptarse a la sociedad (Ackerman 1981). 
La institución familiar, al igual que la sociedad, ha sufrido múltiples cambios propiciados por los patrones de trabajo, de entretenimiento y avances tecnológicos, lo cual provoca que se esté expuesto a mayor información, nuevos y diferentes estilos de vida, ideologías y valores. Según Gergen (1991), existe un incremento de nuevos acuerdos para vivir juntos, lo que implica que cada vez la definición de familia y sus funciones esté cambiando.

La percepción que cada individuo tiene de su familia está conformada por su propia percepción sobre las relaciones entre los miembros de la misma y por la integración del conjunto de las percepciones de cada uno de sus integrantes. De ahí que lo que interioriza es la familia como un sistema formado por interrelaciones en el tiempo y en el espacio (Laing, 1986). Donde la comunicación adecuada y abierta entre los miembros es una condición necesaria en las relaciones familiares, funcionamiento y satisfacción.

Se ha establecido que la relación con ambos padres y la comunicación con ellos, así como el control que ejercen sobre los hijos está relacionada directamente con la autoestima y seguridad de los adolescentes (Neighbors, Forehand \& Vicar, 1993; Brage \& Meredith, 1994; Lackovic, Dekovik \& Opacic, 1994). En esta dirección, se puede decir que la comunicación es un factor de influencia sobre la conducta futura en los diversos ámbitos en los que el joven se desenvuelve.

Es así que la calidad de las relaciones entre padre e hijos se asocia a los estilos de vida que se observan en los adolescentes. Las relaciones con la madre son importantes, pero también son cruciales las relaciones con el padre. Según Rodrigo, Máiquez, García, Martínez y Martín (2004), es crucial la disponibilidad del padre, su implicación en los asuntos del hijo o hija, su capacidad de comunicación y el grado de apoyo paterno que percibe el adolescente para sentirse satisfecho con su entorno.

En su estudio, Álvarez (1999) confirma esta relación al señalar la importancia del padre en la satisfacción de la adolescente en su vida familiar, ya que cuando existe una buena comunicación con él la satisfacción es alta, lo que no sucede con la madre. Al parecer, entonces en la relación padrehija gravita más la influencia del padre y, por lo tanto, la crisis propia de esa edad disminuye. La influencia de la madre pareciera tener un menor peso, dado que las relaciones madre-hija, por lo general, son mejores y no se diferencian mucho entre hombres y mujeres.

Continuando con los estilos parentales, según Díaz-Guerrero (1994), el rol tradicional del padre en la cultura mexicana ha sido considerado básicamente como fuente de autoridad, disciplina y apoyo económico. Otros (Neighbors et al., 1993; Lackovic et al., 1994) enfatizan el rol de la madre como cariñoso y comprensivo. Es así que "los adolescentes que se sientes felices, simpáticos, que le caen bien a la gente y que se sienten seguros de sí mismos, son los que perciben mayor apoyo, comunicación y aceptación tanto de su papá como de su mamá y además perciben a sus padres comprensivos, amigables, amorosos, buenos, cariñosos y justos" (Andrade \& Díaz-Loving, 1997:204).

Las relaciones familiares se harán más sólidas si se establece una buena comunicación, por el contrario, si los canales de comunicación se encuentran bloqueados de manera impositiva habrá una resistencia a cambiar, dando como resultado el desarrollo de un síntoma que pudiera alterar el desarrollo de los hijos. El INEGI y la Secretaría de Educación Pública, a través del Instituto Nacional de la Juventud, llevaron a cabo una encuesta de gran importancia para el país en el 2000. Entre los datos significativos que se encontraron parece que los jóvenes en México mantienen gran apego e identificación familiar. Los padres ejercen una reconocida autoridad sobre sus hijos y la madre constituye el principal apoyo moral (INEGI, 2000).

Otros datos reportados por esta encuesta indican que del total de jóvenes que permanecen en el hogar paterno, $66 \%$ no planea dejarlo, porque se encuentra a gusto en su casa. El $40 \%$ realiza las actividades de diversión en compañía de su madre. Ante la presencia de problemas, $46 \%$ de los jóvenes platica siempre con su madre y $42 \%$ lo hace sólo en algunas ocasiones. Al padre se le consulta con menor frecuencia, y 47\% de los jóvenes nunca recurre a él. En opinión de $66 \%$ de los jóvenes, ante una acción de conducta inadecuada los padres reaccionan platicando con ellos; al 18\% les imponen un castigo, ya sea estableciendo restricciones, golpeándolos, dejándoles de hablar o insultándolos, y al $11 \%$ se le muestra una actitud indiferente. Los jóvenes reconocen en la familia el espacio donde 
se recibe y se da afecto. El 91\% declara tener buen trato con la madre y el $81 \%$ con el padre. De esta forma, $30 \%$ de ellos, reconoce como principales valores de sus padres, el apoyo y la solidaridad con los integrantes de la familia; $24 \%$, la responsabilidad y su papel como proveedores; $16 \%$, el respeto hacia sus decisiones, y $23 \%$ las manifestaciones de afecto (INEGI, 2000).

Es importante señalar que los patrones de conducta que existen en la familia son dinámicos y pueden ser confrontados y adaptados a los cambios requeridos por las diferentes etapas que cursa el ciclo vital de la familia. Estas etapas son momentos de tensión y crisis, que deberán comunicarse y replantear una nueva situación, ya que la familia es un sistema en constante transformación, tal como lo señala Andolfi (1984).

La solución de este conflicto familiar dependerá del tipo de interacción que se dé entre los miembros de la misma, así como de la atención que los padres brinden a los hijos. En este sentido la comunicación entre padres e hijos ha demostrado ser un factor protector o de riesgo.

El término estrés se refiere a una respuesta del cuerpo a agentes que resultan amenazantes o desadaptativos. Fue definido por Lazarus y Folkman (1984) como "una relación entre la persona y el medio ambiente, que es evaluada por la persona como agotador -taxing-, que excede sus recursos poniendo en riesgo su bienestar" (Lazarus \& Folkman, 1984:19), y son ellos los que a través de su Teoría Transaccional del Estrés proponen que lo que importa no es el estresor en sí, sino la manera de evaluarlo y afrontarlo. Así mismo, Sandín (2003) habla de un estrés social y apunta que los diferentes estresores están interrelacionados y que las variables sociales (como el apoyo) influyen en la manera de afrontar el estrés, inclusive se han señalado formas de afrontamiento como la autoestima, el optimismo, la autoeficacia, por mencionar algunos.

En la literatura se ha encontrado una asociación entre estrés y satisfacción con la vida, como lo muestra un estudio transcultural realizado por Matheny, Roque y Curlette (2008), quienes detectaron una correlación negativa entre estrés percibido y satisfacción con la vida. Además de no encontrar diferencias entre hombres y mujeres en función de la satisfacción con la vida y el estrés percibido, hallazgo que coincide con los datos reportados por Reig-Ferrer et al., (2000), quienes señalan que el grado de satisfacción con la vida no guarda relación con la edad y sexo. Empero, estos últimos plantean que sí existe una asociación significativa con el índice de satisfacción con los estudios, el estado de salud en el último año, el malestar físico y psicológico. Refieren que a mayor satisfacción con la vida, menor malestar, estrés reciente, estrés asociado a estudios y estrés en otros dominios de la vida. Así, Reig-Ferrer et al. (2000) concluyen que el estrés es inversamente proporcional a niveles de satisfacción con la vida y ésta presenta asociación con la calidad de vida.

Por otro lado, en una investigación con estudiantes universitarios de Monterrey, México, se encontró que al enfrentar cambios y requerimientos de su ambiente (estresores), éstos pueden llegar a desencadenar síntomas que afectan la calidad de vida de la persona, tales como fatiga, dolor de estomago, de pecho, cefalea, trastornos gastrointestinales, entre otros (González \& Landero, 2008a). Los autores agregan que si el estudiante no tiene los recursos suficientes para afrontar los estresores su rendimiento académico puede verse seriamente afectado; este estudio también reportó que las mujeres presentan niveles de estrés más elevados que los hombres.

Finalmente, Monzon (2008) reportó que ante situaciones de exámenes el nivel de estrés en los estudiantes tiende a aumentar. De la misma forma González y Landero (2008b) reportan en sus hallazgos en su estudio con población universitaria que el grado de estrés detectado es similar al encontrado en muestras de personas con enfermedades crónicas, concluyendo así que es necesario ahondar más en éste fenómeno.

Tomando en cuenta lo expuesto anteriormente, el objetivo del presente estudio pretende obtener información sobre la relación existente entre la satisfacción con la vida, la comunicación con los padres y el nivel de estrés de los jóvenes universitarios de tres ciudades del noreste de la República Mexicana, a saber, Monterrey, Nuevo León, Saltillo, Coahuila y Tampico, Tamaulipas. En donde de acuerdo con algunos autores se esperaría que entre mejor comunicación con los padres, pudiera existir mayor satisfacción con la vida y menor estrés. 


\section{Método}

Determinar las diferencias y relación en los niveles de estrés percibido, satisfacción con la vida y comunicación con el padre y madre, entre ciudades y género en jóvenes universitarios.

\section{Participantes}

La muestra de este estudio quedó conformada por 482 estudiantes de licenciatura en psicología de tres ciudades de la zona noreste de México (Monterrey Nuevo León, Tampico Tamaulipas y Saltillo Coahuila.), todos ellos solteros. La descripción de la muestra se presenta a detalle en la Tabla 1.

Tabla 1. Descripción de las muestra por ciudad

\begin{tabular}{ccccc}
\hline Ciudad & \multicolumn{2}{c}{ Participantes } & \multicolumn{2}{c}{ Edad } \\
\cline { 2 - 5 } & Hombres & Mujeres & Promedio & DE \\
\hline Monterrey & 49 & 148 & 18.93 & 1.51 \\
Tampico & 31 & 132 & 20.01 & 1.47 \\
Saltillo & 42 & 80 & 19.77 & 1.86 \\
\hline
\end{tabular}

\section{Instrumentos}

La Escala de Estrés Percibido (PSS). Se utilizó la adaptación a la población mexicana de la escala de estrés percibido de Cohen, Kamarak y Mermelstein (1983, en González \& Landero, 2007). Esta escala de tipo Likert está compuesta por 14 reactivos, de los cuales siete se codifican a la inversa $(4,5,6,7,9,10$ y 13). Pretende medir el nivel de estrés que la persona percibe a partir de su medio ambiente ante situaciones que el participante puede controlar, así como situaciones que no puede controlar. Esta escala presenta un índice de confiabilidad $\alpha$ de Cronbach de .831 en este estudio, concordando con lo encontrado por González y Landero (2007).

Subescala de comunicación. Se tomó la subescala de comunicación con el padre, a partir del cuestionario de comunicación familiar de Andrade (1998). Esta subescala trata de medir el nivel de comunicación a partir de aspectos como la confianza que tiene los hijos con el padre o madre. También analiza aspectos como el nivel de satisfacción del padre hacia con el hijo (se refiere a la percepción que tiene el hijo sobre esto). Esta subescala está compuesta por 6 reactivos de tipo Likert. Debido a esta división no se tienen valores de alfa previos. La subescala presenta en la comunicación con el padre y la madre índices de consistencia interna $\alpha$ de Cronbach de .931 y .915 , respectivamente.

Escala de satisfacción con la vida. También se utilizó una escala para medir el nivel de satisfacción que el estudiante tiene con su vida. Esta escala es una adaptación que llevaron a cabo Atienza, Pons, Balaguer y García-Merita (2000), a partir de la escala original propuesta por Diener, Emmons, Larsen y Griffin (1985). Esta escala está compuesta por 5 reactivos de tipo Likert, en donde se le pregunta a la persona acerca del nivel de satisfacción con las cosas, situaciones que tiene en su vida general. Esta escala ha presentado un índice de confiabilidad $\alpha$ de Cronbach de .82 y .76 respectivamente (Atienza, Pons, Balaguer \& García-Merita, 2000; Montoya \& Landero, 2008).

\section{Procedimiento}

Como primera fase se llevó a cabo la aplicación de las escalas y subescalas arriba mencionadas. Estas se aplicaron en las aulas de las facultades participantes, durante turnos regulares de clases. Esto nos indica que al menos algunos factores que pudieran funcionar como distractores se controlaron y que la información propiciada por el encargado de la aplicación tuvo más probabilidad de ser 
atendida por los alumnos. Al momento de la aplicación se les informó a los alumnos que su participación era voluntaria y que no tenía ninguna repercusión en sus calificaciones escolares. Además, se les informó que los datos se tratarían de manera confidencial y que solo las personas vinculadas con la investigación tendrían acceso a ellos. A los participantes que aceptaron, se les pidió que contestaran lo más honestamente posible.

Como segunda fase, se llevó a cabo la codificación de los datos, cada encuesta recibió un número de folio con el cual se pudo llevar a cabo la verificación de los datos y por lo tanto de la buena construcción de la base de datos con la cual se trabajó.

\section{Análisis de datos}

Se determinó la normalidad de la distribución utilizando la prueba Kolmogorov-Smirnov, en base a los resultados se tomó la decisión de utilizar pruebas no paramétricas congruentes con la distribución y el objetivo del estudio.

\section{Resultados}

Se obtuvieron los análisis de confiabilidad de las escalas utilizadas en la muestra estudiada. La Escala de Estrés Percibido presentó un índice de confiabilidad $\alpha$ de Cronbach de .831, las Subescalas de comunicación presentaron en la comunicación con el padre y la madre índices de consistencia interna $\alpha$ de Cronbach de .931 y .915 respectivamente. Por último la Escala de satisfacción con la vida presentó un índice de confiabilidad $\alpha$ de Cronbach de .823. Todos estos índices corroborando una confiabilidad adecuada de todos los instrumentos.

Se llevó a cabo la prueba de Kolmogorov-Smirnov para comprobar la normalidad de los datos de las sumatorias de las escalas de estrés percibido, satisfacción con la vida y comunicación con padre y madre. $\mathrm{Al}$ encontrar que los datos no tienen una distribución normal, se decidió utilizar pruebas no paramétricas.

Para el primer análisis se realizó una comparación de grupos entre hombres y mujeres en base a las variables de estudio utilizadas, se usó el estadístico U de Mann-Whitney, no se encontraron diferencias por sexo en ninguna de las variables, en ninguna de las ciudades, a continuación se detallan los datos en la tabla 2.

Tabla 2. Resultados de la comparación entre hombres y mujeres en la muestra

\begin{tabular}{cccccc}
\hline Ciudad & Estadístico & Estrés & Satisfacción & $\begin{array}{c}\text { Comunicación } \\
\text { papá }\end{array}$ & $\begin{array}{c}\text { Comunicación } \\
\text { mamá }\end{array}$ \\
\hline Monterrey & $\begin{array}{c}\text { Mann-Whitney } \\
\mathrm{U}\end{array}$ & 512.500 & 1760.500 & 1578.000 & 1817.000 \\
& $\mathrm{Z}$ & -.456 & -1.004 & -1.537 & -.343 \\
Tampico & Mann-Whitney & 338.000 & 1750.000 & 1940.500 & 1771.000 \\
& $\mathrm{U}$ & & & & -.928 \\
& $\mathrm{Z}$ & -1.469 & -1.201 & -.320 & 1304.500 \\
Saltillo & Mann-Whitney & 270.500 & 1391.500 & 1487.000 & -1.838 \\
\hline & $\mathrm{U}$ & -1.668 & -1.566 & -.076 & \\
\hline
\end{tabular}

Nivel de significancia menores a $0.05=* \quad$ Nivel de significancia menores a $0.01=* *$ 
Por otra parte se utilizó la prueba Kruskal-Wallis, en donde se detectó que sí hay diferencias significativas entre las ciudades, pero solamente en las variables de estrés y satisfacción y sólo en las mujeres (ver tabla 3).

Tabla 3. Resultados de la prueba Kruskal-Wallis

\begin{tabular}{cccccc}
\hline Sexo & Estadístico & Estrés & Satisfacción & $\begin{array}{c}\text { Comunicación } \\
\text { papá }\end{array}$ & $\begin{array}{c}\text { Comunicación } \\
\text { mamá }\end{array}$ \\
\hline Hombre & Chi-cuadrado & 1.037 & 4.044 & 5.296 & 3.257 \\
Mujer & Chi-cuadrado & $15.056^{* *}$ & $19.137^{* *}$ & 1.291 & .259 \\
\hline
\end{tabular}

Nivel de significancia menores a $0.01=* *$

Se realizaron correlaciones por rango de Spearman entre las variables con los datos de las diferentes ciudades, encontrándose diversas correlaciones significativas, como se indica en la tabla 4.

Tabla 4. Resultados de correlaciones

\begin{tabular}{|c|c|c|c|c|c|}
\hline Ciudad & & Estrés & S.V. & C.P. & C.M. \\
\hline \multirow[t]{4}{*}{ Monterrey } & Estrés & ------- & & & \\
\hline & S.V. & $-.359^{* *}$ & ------- & & \\
\hline & C.P. & -.145 & $.428 * *$ & ------- & \\
\hline & C.M. & -.183 & $.291 * *$ & $.683 * *$ & ------ \\
\hline \multirow[t]{4}{*}{ Tamaulipas } & Estrés & ------- & $.459 * *$ & -.060 & -.060 \\
\hline & S.V. & $-.459 * *$ & ------- & $.326^{* *}$ & $.387 * *$ \\
\hline & C.P. & -.060 & $.326^{* *}$ & ------- & $.599 * *$ \\
\hline & C.M. & $-.240^{*}$ & $.387 * *$ & $.599 * *$ & ------- \\
\hline \multirow[t]{4}{*}{ Coahuila } & Estrés & ------- & $.684 * *$ & $-.424 * *$ & -.257 \\
\hline & S.V. & $-.684 * *$ & ------- & $.378 * *$ & $.475^{* *}$ \\
\hline & C.P. & $-.424 * *$ & $.378 * *$ & ------- & $.644 * *$ \\
\hline & C.M. & -.257 & $.475^{* *}$ & $.644 * *$ & ------- \\
\hline
\end{tabular}

Nivel de significancia menores a $0.05=* \quad$ Nivel de significancia menores a $0.01=* *$

Satisfacción con la vida = S.V., Comunicación con el padre = C.P., Comunicación con la madre = C.M.

Discusión y conclusiones

Los resultados en este estudio son por demás interesantes. Mediante nuestro estudio se pudo determinar que existe una correlación positiva entre la comunicación con ambos padres y la satisfacción con la vida en las tres ciudades estudiadas, concordando con Andrade y Díaz-Loving (1997). Por el contrario, se detectó solo en la ciudad de Saltillo una correlación negativa entre comunicación con el padre y estrés percibido, y en Tampico una correlación negativa entre comunicación con la 
madre y estrés percibido. Asimismo no se obtuvieron diferencias significativas en la comunicación con ambos padres por género ni por ciudad. Esto pudiera estar asociado a que las poblaciones estudiadas, del noreste de México, puedan tener características semejantes en cuanto a la comunicación en general con sus padres, lo que valdría la pena contrastarlo con poblaciones del centro y sur del país.

Es bien sabido que el padecer un nivel alto de estrés dependerá de los recursos con los que cuenta la persona para hacer frente a las demandas de su entorno (Sandín, 2003). En este estudio, se encontraron diferencias en los niveles de estrés percibido y satisfacción con la vida en las tres ciudades estudiadas (Monterrey, Tampico y Coahuila), pero solo en población femenina. Las mujeres presentaron niveles de estrés más elevados, lo cual concuerda con lo encontrado por González y Landero (2008a), y esto es una evidencia más de que en la población femenina los niveles de estrés suelen ser mayores.

Los niveles de satisfacción con la vida no mostraron diferencias significativas al compararse entre ciudades, pero sí lo hicieron al comparar entre hombres y mujeres, y esta variable, al igual que el estrés fue significativa en la población femenina, esto no es congruente con lo apuntado por Moyano y Ramos (2007). Ellos encontraron que la satisfacción con la vida en las mujeres es menor ya que las expectativas a futuro son más elevadas y los recursos con los que cuentan no son los necesarios para alcanzar esas metas.

Asimismo, en cuanto a las correlaciones entre estrés y satisfacción con la vida, estas fueron significativas y negativas, lo que nos indica que a mayor estrés menor es la satisfacción con la vida. Resultados similares encontraron Reig-Ferrer et al. (2000) y Matheny, Roque y Curlette (2008), aunque estos últimos no encontraron diferencias en el nivel de estrés entre hombres y mujeres y en este estudio sí aparecen.

Consideramos que se debe seguir explorando las variables de estrés y satisfacción con la vida, para poder explicar el porqué de éstos resultados, y también para corroborar si esto se presenta en población generalizada o solamente en estudiantes universitarios de esta zona del país.

\section{Referencias}

Ackerman, N. (1981). Diagnóstico y tratamiento de las relaciones familiares. Argentina: Paidós.

Álvarez, M. (1999). Comunicación padre-hija: Importancia del padre en la adolescencia [en línea]. Chile: Revista Creces. Recuperado el 10 de junio de 2010 de http://www.creces.cl/new/index. asp?imat $=\% 20 \% 20 \% 3 \mathrm{E} \% 20 \% 201 \& \mathrm{tc}=3 \& \mathrm{nc}=5 \& \operatorname{art}=586$

Andolfi, M. (1984). Terapia familiar. México: Paidós.

Andrade, P.P. \& Díaz-Loving, R. (1997). Ambiente familiar y características de personalidad. Revista de Psicología Social y Personalidad, 13 (2), 199-210.

Andrade, P.P. (1998). El ambiente familiar del adolescente. Tesis de Doctorado en Psicología Social. Facultad de Psicología, UNAM. México.

Ardila, R. (2003). Calidad de Vida una Definición Integradora. Revista Latinoamericana de Psicología, 35 (2), 161-164.

Argyle, M. (1992). La psicología de la felicidad. España: Alianza.

Arita-Watanabe, B.Y. (2005). Satisfacción por la vida y teoría homeostática del bienestar. Psicología y Salud, 15 (1), 121-126.

Atienza, F. L.; Pons, D.; Balaguer, I. \& García-Merita, M. (2000). Propiedades psicométricas de la escala de satisfacción con la vida en adolescentes. Psicothema, 12(2), 314-319. 
Brage, D. \& Meredith, W. (1994). A causal model of adolescent depression. The Journal of Psychology, 128 (4), 455-468.

Castro-Solano, A. \& Díaz-Morales, J.F. (2002). Objetivos de vida y satisfacción vital en adolescentes españoles y argentinos. Psicothema, 14(1), 112-117.

Díaz-Guerrero, R. (1994). Psicología del mexicano. Descubrimiento de la etnopsicología. México: Trillas.

Diener, E. \& Eunkook, M.S. (2003). National differences in subjective well-being. En D. Kahneman, E. Diener \& N. Schwartz (eds.): Well-being. The foundations of hedonic psychology (pp. 434450). EUA: Russell Sage Foundation.

Diener, E. D.; Emmons, R. A.; Larsen, R. J. \& Griffin, S. (1985). The Satisfaction With Life Scale. Journal of Personality Assessment, 49, 71-75.

García-Viniegras, C. \& González, I. (2000). La categoría bienestar psicológico, su relación con otras categorías sociales. Revista Cubana de Medicina Integral, 16(6), 586-592.

Gergen, M. (1991). The saturated family. Family Therapy Networker, 15, 26-35.

González, M. T. \& Landero, R. (2008a). Síntomas psicosomáticos y estrés: comparación de un modelo estructural entre hombres y mujeres. Ciencia UANL, 11(4), 403-410.

González, M. T. \& Landero, R. (2008b). Confirmación de un modelo explicativo para estrés y síntomas psicosomáticos mediante ecuaciones estructurales. Revista Panamericana de Salud Publica, 23(1), 7-18.

González, M. T. \& Landero, R. (2007). Factor Structure of the Perceived Stress Scale (PSS) in a Sample from Mexico. The Spanish Journal of Psychology, 10(1), 199-206.

Instituto Nacional de Geografía, Estadística e Informática (2000). Encuesta Nacional de la Juventud. Secretaría de Educación Pública/Instituto Nacional de la Juventud, México.

Lackovic-Grygin, K.; Dekovik, M. \& Opacic, G. (1994). Pubertal status, interaction with significant others and self-esteem of adolescent girls. Adolescence, 29(115), 691-700.

Laing, R. (1986). La familia y la estructura individua. En: La crisis de la familia. México: La red de Jonás.

Lazarus, R. S. \& Folkman, S. (1984). Stress, appraisal and coping. EUA: Springer.

Linton, R. (1972). La historia natural de la familia. En: Horkheimer, M., Fromm, E., Abrams, CH., Schreker, P., Merton, R., Swift, A. (Eds). La familia. España: Península.

Matheny, K.; Roque-Tovar, B.E. \& Curlette, W.L. (2008). Estrés percibido, recursos de afrontamiento y satisfacción con la vida entre estudiantes universitarios de México y Estados Unidos: un estudio transcultural. Anales de Psicología, 24(1), 49-57.

Montoya, B.I. \& Landero, R. (2008). Satisfacción con la vida y autoestima en jóvenes de familias monoparentales y biparentales. Psicología y Salud, 18(1), 117-122.

Moyano, D. \& Ramos, A. (2007). Bienestar subjetivo: midiendo satisfacción vital, felicidad y salud en población chilena de la Región Maule. Revista Universum, 22(2), 1-10.

Neighbors, B.; Forehand, R. \& Mc Vicar, D. (1993). Resilient adolescents and interparental conflict. American Journal of Orthopsychiatry, 63(3), 462-471.

Reig-Ferrer, A.; Cabrero-García, J.; Ferrer-Cascales, R.I. \& Richart-Martínez, M. (2000). La calidad de vida y el estado de salud de los estudiantes universitarios [en línea]. España: Universidad de Alicante/Biblioteca Virtual Miguel de Cervantes. Recuperado el 10 de junio de 2010 de http:// www.cervantesvirtual.com/servlet/SirveObras/00360563299914928537857/index.htm 
RODRIGO CANTÚ / JULYMAR ALEGRE / OSWALDO MARTÍNEZ / MAYRA CHÁVEZ / SISSI ARELLANO / CESAR SAUCEDO / JAVIER TALAMANTES / RENÉ LANDERO

Rodrigo, J.M.; Máiquez, M.L.; García, M.; Mendoza, R.; Rubio, A.; Martínez, A. \& Martín, J.C. (2004). Relaciones padres-hijos y estilos de vida en la adolescencia. Psicothema, 16(2), 203-210.

Sandín, B. (2003). El estrés: un análisis basado en el papel de los factores sociales. International Journal of Clinical and Health Psychology, 3(1), 141-157. 